\title{
Role of stereotactic biopsy in multifocal brain lesions: considerations on 100 consecutive cases
}

Angelo Franzini, Filippo Leocata, Cesare Giorgi, Antonio Allegranza, Domenico Servello, Giovanni Broggi

\author{
Department of \\ Neurosurgery, Istituto \\ Nazionale Neurologico \\ "C Besta", Milano, \\ Italy \\ A Franzini \\ F Leocata \\ C Giorgi \\ A Allegranza \\ D Servello \\ G Broggi \\ Correspondence to: \\ Dr Angelo Franzini, Istituto \\ Neurologico "C Besta", Via \\ Celoria 11, 20133 Milano, \\ Italy. \\ Received 17 February 1993 \\ and in revised form \\ 2 January 1994 \\ Accepted 7 January 1994
}

\begin{abstract}
One hundred patients affected by multifocal brain lesions were investigated by serial stereotactic biopsy. Systemic diseases and primary neoplasms elsewhere were previously ruled out. The histological diagnosis obtained in this series comprises malignant gliomas in $37 \%$ of patients; primary non-Hodgkin's brain lymphoma in 15\%; metastatic brain tumours in $15 \%$ (no evidence of the primary tumour at the time of stereotactic surgery); low grade gliomas in $12 \%$; infective diseases in $10 \%$ (including brain abscesses and multifocal viral encephalitis); and ischaemic lesions in $6 \%$. In addition, two patients with germinomas, two with primitive neuroepithelial tumours, two with multiple telangiectases, and one with a teratoma were also included in this series. Histological findings obtained by stereotactic procedures guided the choice of treatment, avoiding the risks of blind treatments. Indications and future perspectives for stereotactic surgery in multifocal brain lesions are discussed with emphasis on advances in diagnostic and therapeutic tools.
\end{abstract}

(F Neurol Neurosurg Psychiatry 1994;57:957-960)

The use of CT and MRI allows the pattern of multiple brain lesions to be determined in patients with a neurological focal deficit or with symptoms of intracranial hypertension.

Most of these patients could be diagnosed correctly regarding the nature of their intracranial lesions after clinical and laboratory findings as in multiple sclerosis, in secondary infective and parasitic diseases, in metastatic tumours, and in the brain localisations of systemic diseases. Nevertheless, in some cases the multiple lesions detected by CT and MRI remain the only demonstrable evidence of the illness and treatment may be planned only on the presumptive diagnosis.

The usefulness of stereotactic procedures to detect the nature of the lesions and decide treatment is discussed based on our experience of 100 consecutive patients operated on at the National Neurological Institute of Milan.

\section{Materials and methods}

Between 1978 and 1991940 patients underwent stereotactic serial biopsy for brain lesions. One hundred patients out of these series were affected by multiple intracerebral lesions. They were selected for stereotactic biopsy based on the following criteria:

(1) They had no neoplasms outside the nervous system.

(2) They had no systemic diseases possibly related to the brain localisations.

(3) There were no disorders of the cerebral circulation and no evidence of demyelinating diseases of the nervous system.

(4) They had a CT and MRI pattern suggesting different aetiologies in regard to the nature of the lesions.

(5) Neurological and general conditions still allowed a therapeutic choice (Karnofsky stage $>70){ }^{1}$

The ages of these cases ranged from 7 to 66 (mean age 51); 67 were caucasian males. The heralding symptoms included intracranial hypertension in 60 cases, focal neurological deficits in 26 cases, and epileptic seizures in 14 cases. Only in two patients the clinical picture suggested the presence of multiple focal lesions before neuroradiological examinations. Until 1985 the radiological diagnosis was achieved by CT and then by CT and MRI. The number of radiologically demonstrated lesions ranged from two to eight (mean four) in each patient. In 85 patients the lesions were only supratentorial whereas in the other cases the cerebellum and the brainstem were also involved. In 75 cases lesions were localised on both sides. Preliminary corticosteroid treatment $(4 \mathrm{mg}$ dexamethazone thrice daily) was performed in all cases to treat intracranial hypertension and oedema around the lesion, and to rule out primary brain lymphoma.

The stereotactic biopsy was performed with different frames including Riechert, BRW, CRW, Zeppelin, and Leksell systems and since 1986 was assisted by three dimensional computerised reconstruction of CT or MRI images. ${ }^{2-7}$ All tissue specimens were obtained with a Nashold biopsy instrument. Intraoperative smear examinations were performed by methylene blue staining. ${ }^{8-10}$ Two cases with worsening of pre-existing hemiparesis due to a small bleed within the basal ganglia targets were considered to have permanent morbidity. There was no operative mortality in this series.

\section{Results}

Thirty seven patients had malignant multifocal gliomas including anaplastic astro- 
Table Histological diagnoses obtained by serial stereotactic biopsy in 100 consecutive cases affected by multifocal brain lesions

\begin{tabular}{ll}
\hline Histological diagnosis & Patients $(n=100)$ \\
\hline Malignant gliomas & 37 \\
(27 glioblastomas; 10 anaplastic & \\
$\quad$ astrocytomas) & 12 \\
Low grade astrocytomas & 15 \\
Primary non-Hodgkin's lymphomas & 15 \\
Metastatic tumours & 2 \\
Germinomas & 2 \\
Multifocal primitive neuroepithelial & 1 \\
$\quad$ tumours & 5 \\
Teratomas & 3 \\
Primary bacterial abscesses & 2 \\
Viral encephalitis & 2 \\
Progressive multifocal leucoencephalitis & 4 \\
Gliovascular dysplasias: telangiectases & Ischemic lesions
\end{tabular}

* In seven of these patients the tumour developed as malignant glioma and in five as so-called gliomatosis cerebri.

cytomas (10 patients) and glioblastomas (27 patients).

Twelve patients had low grade gliomas. Seven of these showed clinical and neuroradiological evolution as in multifocal malignant gliomas suggesting the unreliability of the histological grading obtained by stereotactic biopsy in this small series. The remaining five cases developed the clinical and neuroradiological picture of slowly and diffusely growing glioma as in so-called "gliomatosis cerebri". ${ }^{11}$

Fifteen patients had primary nonHodgkin's lymphomas; these responded poorly to the preoperative corticosteroid treatment.

Fifteen patients had metastatic tumours suggesting adenocarcinomas without clear histological evidence of the primary tumour site.

Two patients had multifocal germinomas, two had multifocal primitive neuroepithelial tumours and one had multiple teratomas.

Six patients complained of multiple and progressive strokes resembling the clinical history and the neuroradiological picture of multifocal expanding lesions. The stereotactic procedure showed ischaemic lesions in four patients and gliovascular haemorrhagic dysplasias in two cases. ${ }^{12}{ }^{13}$ Infective diseases were Figure $1 \quad$ T1 weighted
gadolinium enhanced axial MRI for a 40 year old male patient. The patient complained only of mild dyslexia. The stereotactic biopsy disclosed progressive multifocal leucoencephalitis and the patient was treated with azathioprine and cytarabine. found in 10 patients including multiple bacterial abscesses in five cases, ${ }^{14}{ }^{15}$ viral multifocal encephalitis in three cases, and progressive multifocal leucoencephalitis in two patients who were HIV negative by enzyme linked immunosorbent assay (ELISA) before the stereotactic procedure. The last two cases later developed AIDS and the serological evidence appeared. ${ }^{16-18}$

The table summarises the histological diagnoses obtained in the whole series.

The choice of treatment was guided by the histological findings. Multifocal malignant gliomas were treated by chemotherapy with cisplatin, carmustine, and etoposide ${ }^{1920}$ and conventional radiotherapy (6000 rads $)^{21-23}$; the survival in these patients ranged between four and 18 (mean nine) months as in patients affected by single malignant non-operated gliomas.

Multiple primitive brain lymphomas were treated by chemotherapy with methotrexate, vincristine, and carmustine ${ }^{24}$ and external radiotherapy; the survival ranged between 16 months and five years (mean three years).

Metastatic tumours were treated by external brain radiotherapy only (6000 rads). These patients were also investigated for the primary tumours outside the nervous system. The origin of brain metastatic tumours was found in only two cases out of 14. Survival ranged between five and 24 (mean seven) months.

Germinomas were treated by radiotherapy; complete clinical and radiological recovery was obtained in one case (four year follow up) whereas in the other patient early dissemination occurred with fatal recurrence of the disease despite the similar histological findings. Multifocal primitive neuroepithelial tumours were treated as malignant gliomas, with poor results. The patient affected by multiple teratoma underwent chemotherapy with platinex, vepesid, and bleomicine, and external radiotherapy to the whole brain and spinal axis with radiological reduction of the lesions; this patient is still disease free at a three year follow up.

Patients with multiple strokes were given pharmacological treatment with ticlopidine after ruling out microembolic peripheral sources in the heart and supra-ortic vessels.

One patient with gliovascular haemorrhagic dysplasias died within one year after the biopsy due to lesion rebleeding; the second patient is still alive but has complained of progressive worsening without major haemorrhage.

Patients with multiple primary cerebral abscesses were treated by stereotactic aspiration of major lesions and repeated intracavitary and systemic antibiotic treatment. ${ }^{14}$ All patients recovered and are still doing well at three to eight year follow up. Patients with multifocal viral encephalitis and by primitive neuroepithelial tumours had fatal outcomes within four months of stereotactic biopsy.

Figures 1-7 show neuroradiological patterns. 


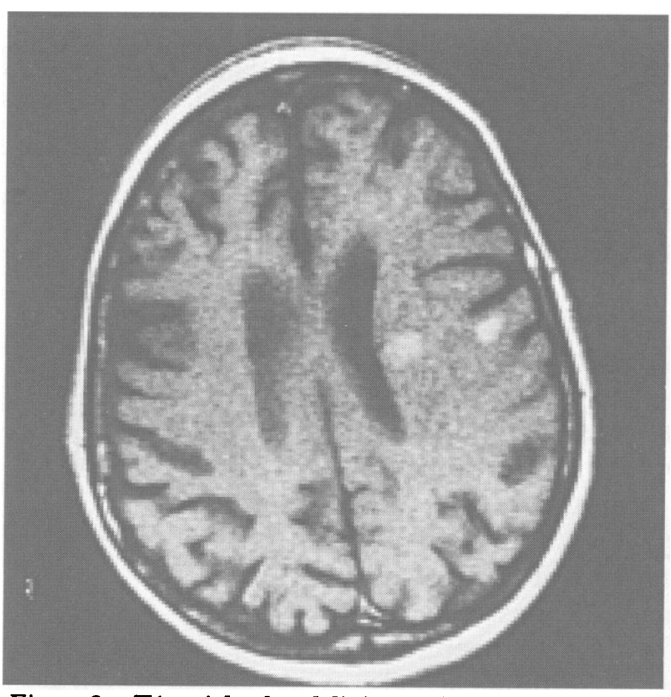

Figure $2 \quad T 1$ weighted gadolinium enhanced axial MRI for a 57 year old patient. The patient complained of drowsiness and quadriparesis. Stereotactic biopsy demonstrated non-Hodgkin's lymphoma and the patient underwent external radiotherapy.

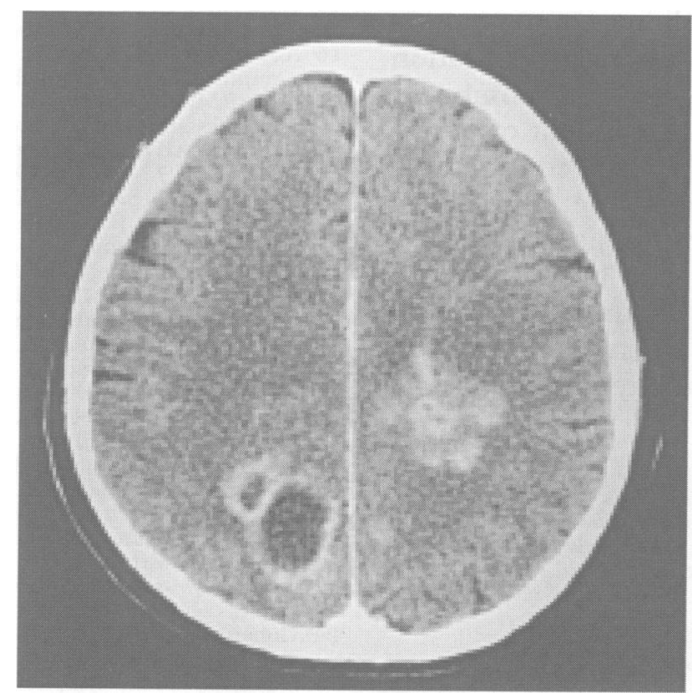

Figure 3 Contrast enhanced CT of a 62 year old male patient. The patient complained of right hemiparesis and aphasia. Stereotactic biopsy disclosed non-Hodgkin's lymphoma and the patient was treated with radiotherapy and chemotherapy (methotrexate, vincristine, and carmustine).

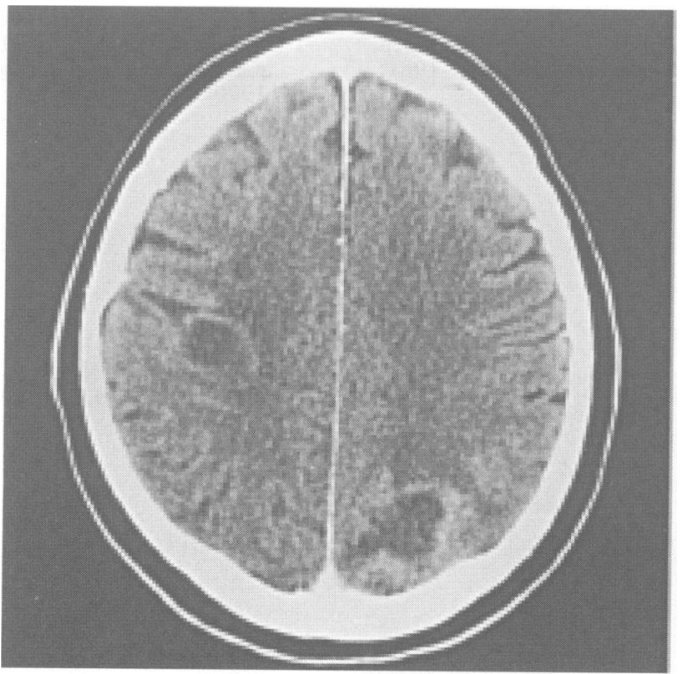

Figure 4 Contrast enhanced CT of a 67 year old male patient. The patient complained of mild left hemiparesis. Stereotactic biopsy demonstrated metastatic tumours and the patient underwent external radiotherapy.

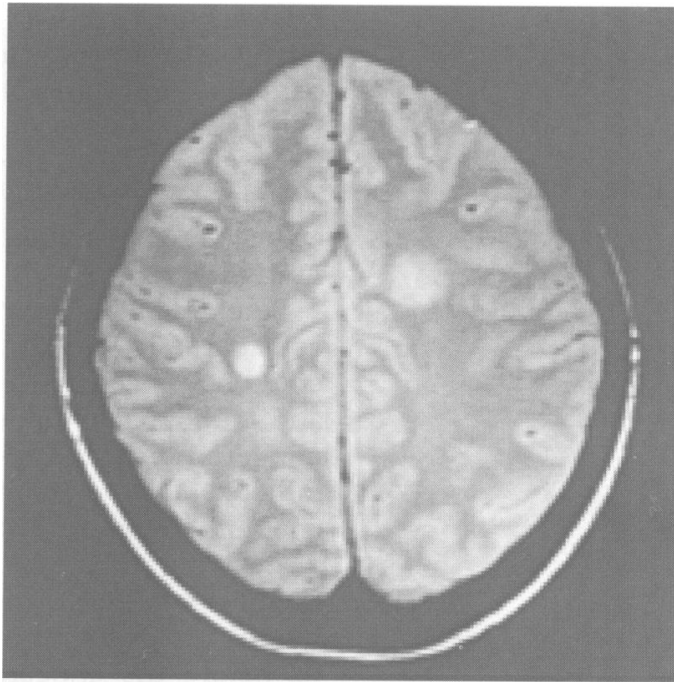

Figure 5 Proton density axial MRI for a 35 year old female patient. The patient complained of headache without focal deficits. Stereotactic biopsy showed a viral multifocal encephalitis and the patient was treated with acyclovir.

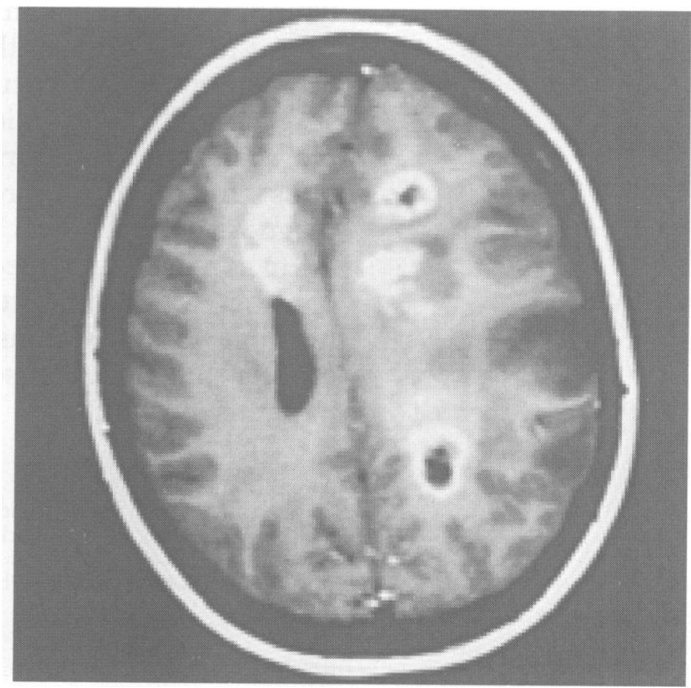

Figure $6 \quad T 1$ weighted gadolinium enhanced axial MRI for a 17 year old female patient. The patient complained of headache, vomiting, and mild right hemiparesis. Stereotactic biopsy demonstrated an anaplastic astrocytoma and the patient underwent chemotherapy (cis-platin, carmustine, etoposide) and conventional radiotherapy.

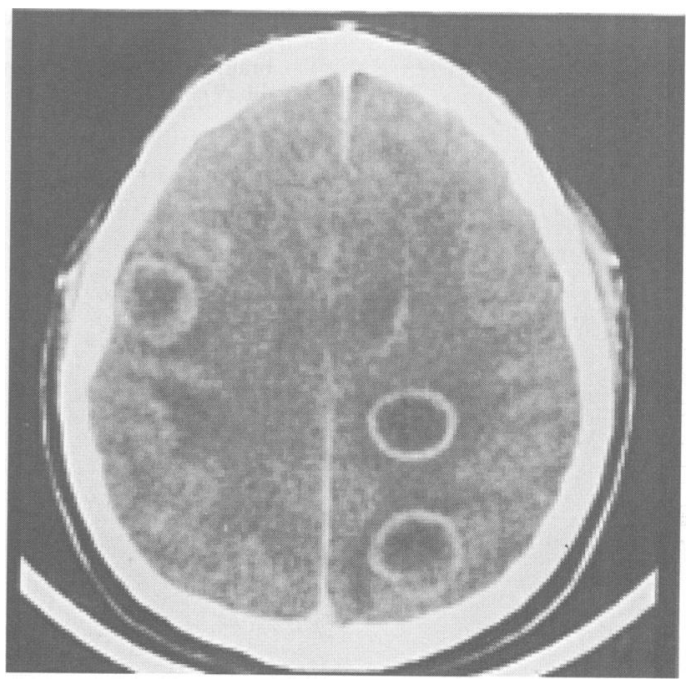

Figure 7 Contrast enhanced CT on a 30 year old male patient. The patient complained of headache and vomiting without focal deficits. Stereotactic biopsy disclosed a pyogenic abscess and the patient was given specific intracavitary and systemic antibiotic treatment. 


\section{Discussion}

The diverse range of diseases in this series of patients emphasises the importance of histological diagnosis to avoid the hazards of treatments based only on clinical and neuroradiological data. The stereotactic biopsy can achieve this goal with low morbidity and zero mortality.

The following points need be taken into consideration. Most non-neoplastic lesions could have been missed because of the clinical and radiological patterns resembling malignant tumours; castastrophic brain damage inflicted by blind radiotherapy and chemotherapy was avoided.

In two patients the diagnosis of AIDS was established after histological findings of brain lesions progressive multifocal leucoencephalitis before the appearance of serological evidence of the disease.

In patients affected by neoplasms the correct histological diagnosis contributed to the evaluation of life expectancy-that is, in brain lymphomas $v$ malignant gliomas.

In the near future, the extensive utilisation of stereotactic radiosurgery and combined surgical and radiosurgical treatment of multiple metastatic tumours ${ }^{25-27}$ will considerably boost the need of preoperative histological diagnosis in multifocal brain lesions.

In conclusion, stereotactic biopsy may be considered the first step in the treatment of multiple brain lesions when the selection criteria that characterise the reported series of patients are fulfilled.

A preliminary report of this study was given at the 10th Congress of the ESSFN, held in Stockholm, Sweden, 2-5 September 1992. The work was partly supported by Associazione "Paolo Zorzi" for Neurosciences, Milano, Italy. We acknowledge Miss Phil Glynn for reviewing the manuscript.

1 Karnofsky DA, Burchenal JH. The clinical evaluation of chemotherapeutic agents in cancer. In: Macleod CM, ed. Evaluation of chemotherapeutic agents. New York: Columbia University Press, 1949:191-205.

2 Apuzzo MLJ, Fredericks CA. The Brown-Roberts-Wells system. In: Lunsford LD, ed. Modern stereotactic neurosurgery. Boston: Nijhoff $M, 1988: 63-77$.

3 Broggi G, Franzini A. Value of serial stereotactic biopsies and impedance monitoring in the treatment of deep brain tumours. $¥$ Neurol Neurosurg Psychiatry 1981; 44:397-401.
4 Giorgi C, Casolino SD, Franzini A, et al. Computer assisted planning of stereotactic neurosurgical procedures. Child Nerv Sys 1989;5:299-302.

5 Kelly PJ, Kall BA, Goerss S. Transposition of volumetric information derived from computed tomography into stereotactic space. Surg Neurol 1984;21:465-71.

6 Lunsford LJ, Martinez AJ. Stereotactic exploration of the brain in the era of computed tomography. Surg Neurol brain in the era

7 Thomas DGT, Nouby RM. Experience in 300 cases of CT-directed stereotaxic surgery for lesion biopsy and CT-directed stereotaxic surgery for lesion biopsy and

8 Eisenhardt $L$, Cushing $\mathrm{H}$. Diagnosis of intracranial tumours by supravital technique. Am f Pathol 1930;6: 541

9 Morris AA. The use of the smear technique in the rapid histological diagnosis of tumour of the central nervous system. F Neurosurg 1947;4:497.

10 Hitchcock E, Morris CS, Sotelo MG, Salmon M. A comparison of smear and imprint techniques for rapid diagparion in Neurol 1986;26:176-82.

11 Couch JR, Weiss SA. Gliomatosis cerebri: report of four cases and review of the literature. Neurology 1976;24: 504-11.

12 Bugiani O. Multiple stroke due to multiple vascular malformation of the brain. Eur Neurol 1984;23:206-10.

13 Testa D, Savoiardo M, Fazio R, Taccagni L, Allegranza A Cerebral multiple glio-vascular displasias; a case report. Clin Neuropathol 1990;9:66-8.

14 Broggi G, Franzini A, Pelucchetti D, Servello D Treatment of deep brain abscesses by stereotactic implantation of an intracavitary device for evacuation and local application of antibiotics. Acta Neurochir (Wien) 1985;76:94-8

15 Gildenberg PL, Franklin PO. Survey of CT-guided stereotactic surgery. Appl Neurophysiol 1985;48:477-80.

16 Astrom KE, Mancall EL, Richardson EP Jr. Progressive multifocal leucoencephalopathy. Brain 1958;81:93.

17 Levy RM, Bredesen DE, Rosenblum ML. Neurological manifestations of the acquired immune deficiency syndrome (AIDS): Experience at UCSF and review of the drome (AIDS): Experience at UCSF and

18 Snider WD Simpson DM, Nielsen S, Gold JWM ider Metroka CE, Posner JB. Neurological complications of the acquired immune deficiency syndro

19 Boiardi A, Silvani A, Milanesi I, Botturi M, Broggi G. Primary glial tumor patients treated by combining cis-platin and etoposide. $\mathcal{F}$ Neurooncol 1991;11:165-70.

20 Kornblith P, Walker M. Chemotherapy for malignan gliomas. F Neurosurg 1988;68:1-17.

21 Marsa GW, Goffinet DK, Rubenstein LJ, Bagshaw MA Megavoltage irradiation in the treatment of gliomas of the brain and spinal cord. Cancer 1975;36:1681-9.

22 Morantz RA. Radiation therapy in the treatment of cerebral astrocytomas. Neurosurgery 1987;20:975-82.

23 Leibel S, Sheline G. Radiotherapy in the treatment of cerebral astrocytoma. In: D Thomas ed. Neurooncology: primary malignant brain tumours. Baltimore, oncology: primary malignant brain tumours.

24 Cohen IJ, Vogel R, Matz S, et al. Successful nonneurotoxic therapy (without radiation) of a multifocal neurotoxic therapy (without radiation) of a multifocal primary brain lymphoma with a methotrexate, vin57:6-11.

25 Adler JR, Cox RS, Kaplan I, Martin DP. Stereotactic radiosurgical treatment of brain metastases. $\mathcal{f}$ Neurosur 1992;76:444-9.

26 Kilstroem L, Karlsson B, Lindquist C, Noren G, Rahn T. Gamma knife surgery for cerebral metastasis. Acto Neurochir (Wien) 1991;52 (suppl):87-9.

27 Loeffler JS, Koy HM, Wen PY, Fine HA, Cheng CW, Mannarino EG, et al. The treatment of recurrent brain metastases with stereotactic radiosurgery. $7 \mathrm{Clin}$ Oncol 1990;8:576-82. 